\title{
Fibrolipoma intermuscular de cadera
}

\section{Intermuscular fibrolipoma of the hip}

\author{
Alejandro Miravete Gálvez, ${ }^{\star}$ Kenya Magally Estrada Zaldivar, ${ }^{\ddagger}$ Javier Camacho Galindo*
}

Citar como: Miravete GA, Estrada ZKM, Camacho GJ. Fibrolipoma intermuscular de cadera. An Med ABC. $2021 ; 66$ (2): $128-131$. https://dx.doi.org/10.35366/100482

\begin{abstract}
RESUMEN
Los lipomas subfasciales intermusculares representan menos de $2 \%$ de todos los lipomas, mientras que la incidencia de los fibrolipomas intermusculares es desconocida, con únicamente reportes aislados y pequeñas series de casos encontrados en la literatura. Presentamos el caso de un paciente femenino de 45 años, quien inició su padecimiento aproximadamente tres semanas previas a su ingreso con dolor insidioso en región inguinal izquierda, sin irradiaciones, de tipo opresivo con una intensidad 3/10 en la EVA, el cual aumenta con el inicio de la deambulación 5/10 en la EVA. En la exploración física se encuentra tumoración de aproximadamente $5 \times 3$ $\mathrm{cm}$ en región anterolateral del muslo, de consistencia suave, adherido a planos profundos, mínimo dolor a la palpación media y profunda. Se realizó resonancia magnética de muslo, se encontró una masa lobular y tejido fibroso que atraviesan dichos lóbulos de aproximadamente $6.4 \times 4.4 \mathrm{~cm}$, con aumento de intensidad de la señal en T1. Se realizó la resección quirúrgica de la lesión y fue enviada a patología quirúrgica con un diagnóstico definitivo de fibrolipoma intermuscular. La baja frecuencia de esta patología hace de interés su reporte y resulta de vital importancia conocer el adecuado abordaje diagnóstico y terapéutico de la lesión.
\end{abstract}

Palabras clave: Cadera, intermuscular, fibrolipoma, resección.

\footnotetext{
* Cirujano Ortopedista y Traumatólogo.

‡ Médico Cirujano.
}

Centro Médico ABC. México.

Recibido: 03/04/2021. Aceptado: 03/06/2021.

Correspondencia: Alejandro Miravete Gálvez

E-mail: dr.miravete@gmail.com

Abreviaturas:

$E V A=$ Escala visual analógica del dolor.

RMN = Resonancia magnética nuclear.

TAC = Tomografía axial computarizada.

\begin{abstract}
Intermuscular subfascial lipomas represent less than $2 \%$ of all lipomas. The specific incidence of intermuscular fibrolipomas is unknown, with only case reports and small series of cases found in the literature. We present the case of a 45-year-old female patient whose current disease initiated approximately three weeks prior to her hospitalization, presenting with an oppressive pain in the inguinal region, without irradiations, with an intensity of 3/10 on the VAS which is exacerbated with mild physycal activity. Physical examination demonstrates a tumor size by approximately $5 \times 3 \mathrm{~cm}$ in the anterolateral region of the muscle, of smooth consistency, adhering to deep planes, with mild pain at medium and deep palpation. An MRI of the muscle was done, a lobular mass was founded of approximately $6.4 \times 4.4 \mathrm{~cm}$ traversed with fibrous strands thick lobes, in intensity of the wave at $T 1$. We realize a surgical resection of the lesion with the final piece sent to pathology for a definitive diagnosis of intermuscular fibrolipoma. The low incidence of this pathology makes it of interest for its report, it is of utter importance to update its accurate diagnostic approach and treatment strategies.
\end{abstract}

Keywords: Hip, intermuscular, fibrolipoma, resection.

\section{INTRODUCCIÓN}

Los lipomas se definen como masas blandas de células adiposas que a menudo se encuentran encapsuladas por una capa fina de tejido fibroso. ${ }^{1,2}$ Son considerados los tumores benignos de tejidos blandos más comunes, con una prevalencia de 2.1 por cada 1,000 habitantes y representan aproximadamente $50 \%$ de todos los tumores del sistema musculoesquelético. ${ }^{3-5}$ Pueden localizarse en cualquier región del cuerpo y pueden clasificarse en superficiales o profundos (sub- 
fasciales). Tienen clara preferencia por el tejido celular subcutáneo, suelen ser pequeños y pesar unos cuantos gramos. De manera común los encontramos en el muslo, hombro y tronco. ${ }^{3,6}$

En contraparte los lipomas subfasciales intermusculares representan menos de $2 \%$ de todos los lipomas. Con respecto a los fibrolipomas intermusculares (un subtipo de lipoma, el cual se origina del septum intermuscular y presenta hipertrofia de adipocitos maduros y tejido fibroso conectivo) su etiología es desconocida, lo cual los hace extremadamente raros con únicamente reportes aislados y pequeñas series de casos encontrados en la literatura. $5,7,8$

La baja frecuencia de esta patología hace de interés su reporte y resulta de vital importancia conocer el adecuado abordaje diagnóstico y terapéutico de la lesión.

\section{CASO CLÍNICO}

Presentamos el caso de un paciente femenino de 45 años, sin antecedentes médicos de importancia. Inició su padecimiento aproximadamente tres semanas previas a su ingreso con dolor insidioso en región inguinal izquierda, sin irradiaciones, de tipo opresivo con una intensidad 3/10 en la escala visual analógica del dolor (EVA), el cual aumenta con el inicio de la deambulación 5/10 en la EVA y cede de manera parcial con el reposo, sin antecedente previo de trauma. Acude con médico general, quien inicia tratamiento antiinflamatorio y relajante muscular con meloxicam y carisoprodol por 14 días sin respuesta al mismo. La paciente refiere aumento de volumen y dolor progresivo en cadera afectada, por lo que decide acudir a consulta con médico ortopedista.

En la exploración física se encuentra tumoración de aproximadamente $5 \times 3 \mathrm{~cm}$ en región anterolateral del muslo, de consistencia suave, adherido a planos profundos, no genera tensión ni cambios de coloración en la piel, no pulsátil, mínimo dolor a la palpación media y profunda.

Se solicitó resonancia magnética nuclear $(\mathrm{RMN})$ de muslo izquierdo, se encontró una masa lobular y tejido fibroso que atraviesan dichos lóbulos de aproximadamente $6.4 \times 4.4 \mathrm{~cm}$, con aumento de intensidad de la señal en T1 sin infiltración del tejido muscular circundante (Figura 1). Por los hallazgos clínicos y de imagen se decidió realizar resección quirúrgica de la lesión.

Se accedió a la lesión a través de un abordaje anterior de cadera, el cual se inició dos centímetros lateral y ligeramente caudales a la espina iliaca anterosuperior con una extensión de aproximadamente $10 \mathrm{~cm}$. Se incidió piel y tejido celular subcutáneo y se desarrolló el intervalo de Hueter conformado por el músculo tensor de la fascia lata y el sartorio, donde se encontró una tumoración blanda ovoide, multilobulada de coloración amarilla, adherida a la fascia del sartorio sin invasión de la cápsula articular, ni de vasos sanguíneos importantes. La tumoración fue resecada en bloque mediante disección roma, cuidando estructuras neurovasculares adyacentes (nervio femorocutáneo lateral y vasos femorales).

Se envió el tejido recolectado al Servicio de Patología Quirúrgica para su diagnóstico definitivo. $\mathrm{Al}$ examen macroscópico se encuentra masa amarilla multilobulada con áreas rosa claro de $78 \mathrm{~g}$ que mide $8.8 \times 6.8 \times 2.1 \mathrm{~cm}$ (Figura 2 ).

Microscópicamente se encontró lesión formada por tejido adiposo maduro, dividido por septos fibrosos gruesos y delgados vascularizados. Sin cambios de tipo neoplásico maligno. Con un diagnóstico definitivo de fibrolipoma maduro (Figura 3).

\section{DISCUSIÓN}

Los lipomas son considerados los tumores benignos de tejidos blandos más comunes, se presentan en pacientes entre los 40 y 60 años y no existe distinción entre sexos.

Debido a la baja incidencia, los lipomas intermusculares pueden ser fácilmente pasados por alto, para diferenciarlos de otros tumores de tejidos blandos es ne-

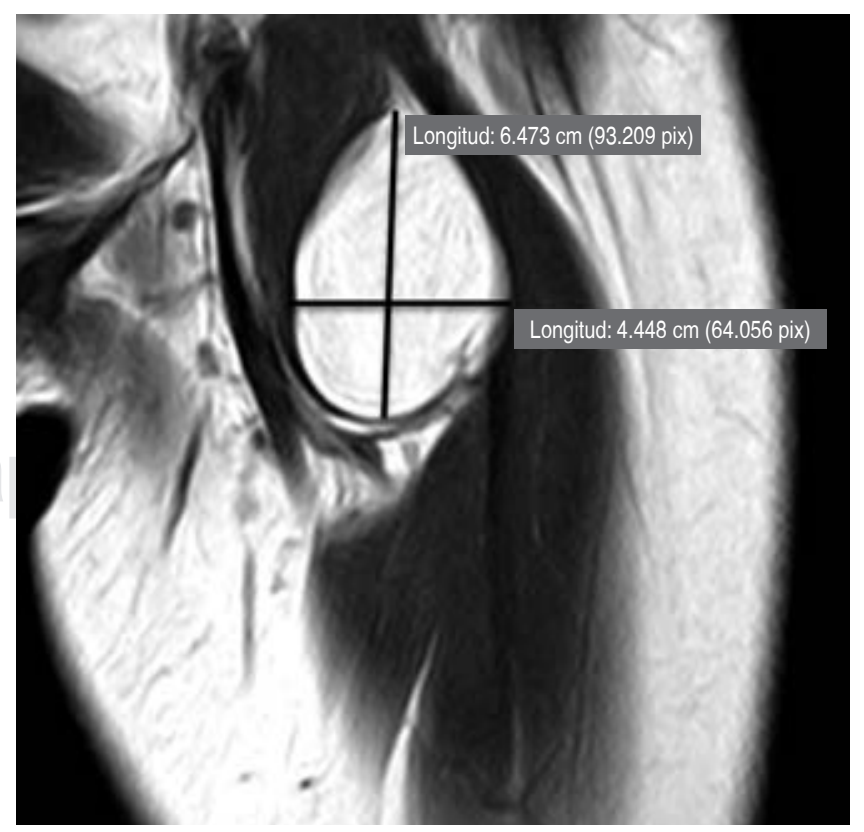

Figura 1: Resonancia magnética nuclear de muslo izquierdo. 

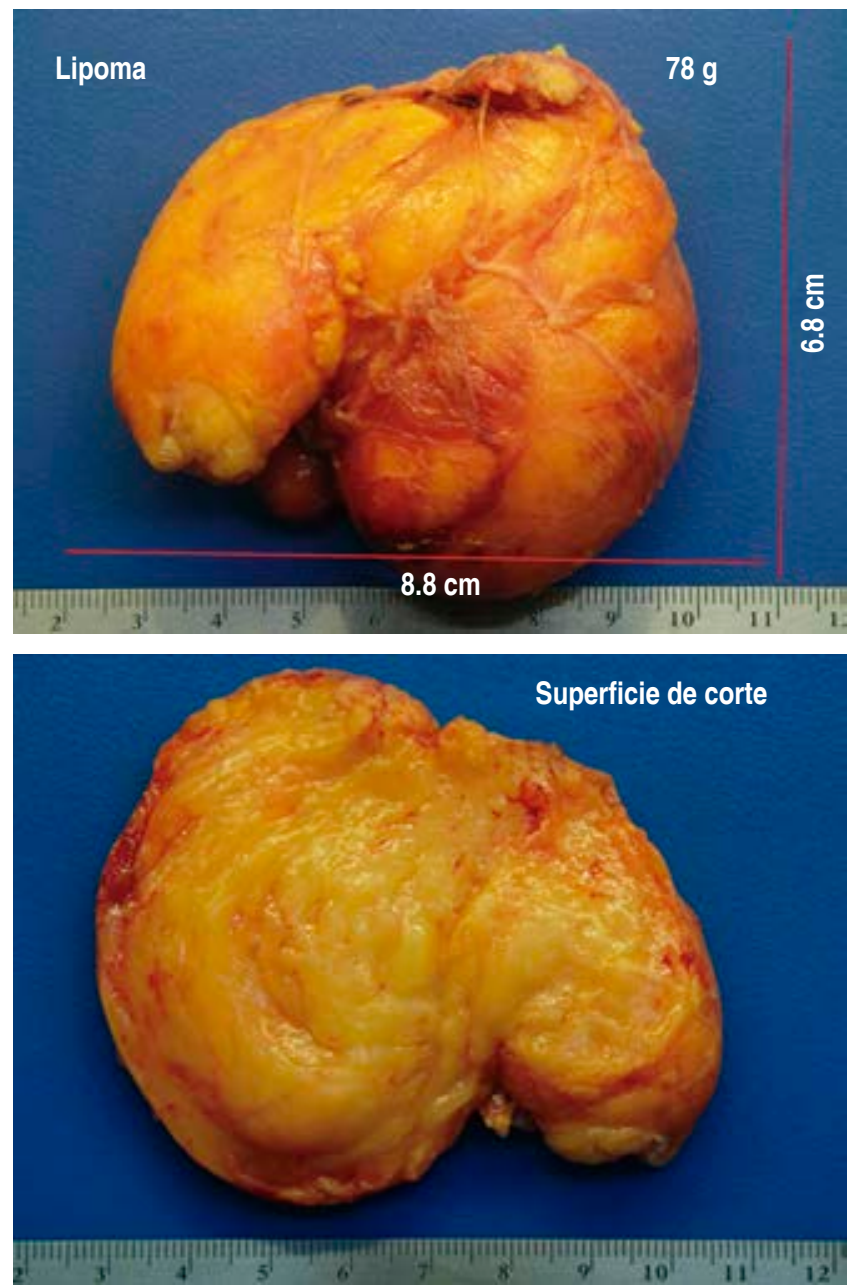

Figura 2: Pieza quirúrgica.

cesario realizar estudios de imagenología complementarios como pueden ser el ultrasonido y la tomografía axial computarizada (TAC). De manera habitual, la mayoría de los lipomas son inicialmente diagnosticados mediante el uso de ultrasonido debido a la disponibilidad y bajo costo de esta herramienta, cuando es necesario resecar la lesión se recomienda ampliamente realizar una resonancia magnética, no sólo para confirmar el diagnóstico, que suele ser fuertemente sugerido por el ultrasonido y/o la tomografía computarizada, sino también por ser el mejor estudio para evaluar las posibles características atípicas que pudieran sugerir un liposarcoma. A su vez, la RMN nos ayuda a dilucidar con mayor exactitud la relación de la tumoración con la anatomía circundante, lo cual es de gran ayuda para la planeación de la resección quirúrgica. ${ }^{9-11}$

Las indicaciones para la resección quirúrgica de este tipo de lesiones incluyen masas subfascia- les mayores a $5 \mathrm{~cm}$, que se encuentren en continuo crecimiento y/o se asocien con dolor; otra indicación relativa, pero de suma importancia, es la apariencia estética que usualmente preocupa a los pacientes. ${ }^{12,13}$

La resección total de las lesiones suele no presentar complicaciones, ya que de manera habitual se encuentra bien delimitada por una pseudocápsula, la cual facilita la disección de ésta.

Posterior a su resección los lipomas intermusculares tienen una recurrencia que varía ampliamente en la literatura entre 1 y $62 \%$, sin datos encontrados para los fibrolipomas intermusculares. La principal causa de recurrencia es la resección incompleta de la lesión. Se ha reportado que cuando esto sucede, la masa solía tener una cercana relación con estructuras neurovasculares adyacentes que no fueron detectadas de manera preoperatoria al no contar con estudios de imagen avanzados (TAC/RMN). Debido a su poca capacidad de malignización los cirujanos optaron por una resección incompleta buscando evitar posibles complicaciones perioperatorias graves. ${ }^{3,12}$

Es importante conocer la histopatología de estas lesiones, ya que es usual que los pacientes se presenten con el ortopedista, quien deberá tomar decisiones para el diagnóstico así como de tratamiento. Los estudios que arriba se mencionan tienen la finalidad de descartar otras lesiones y así tomar la decisión quirúrgica más adecuada. La situación anatómica donde se localiza así como la descripción de la tumoración son indispensables para la toma de decisión terapéutica, es imperativo descartar

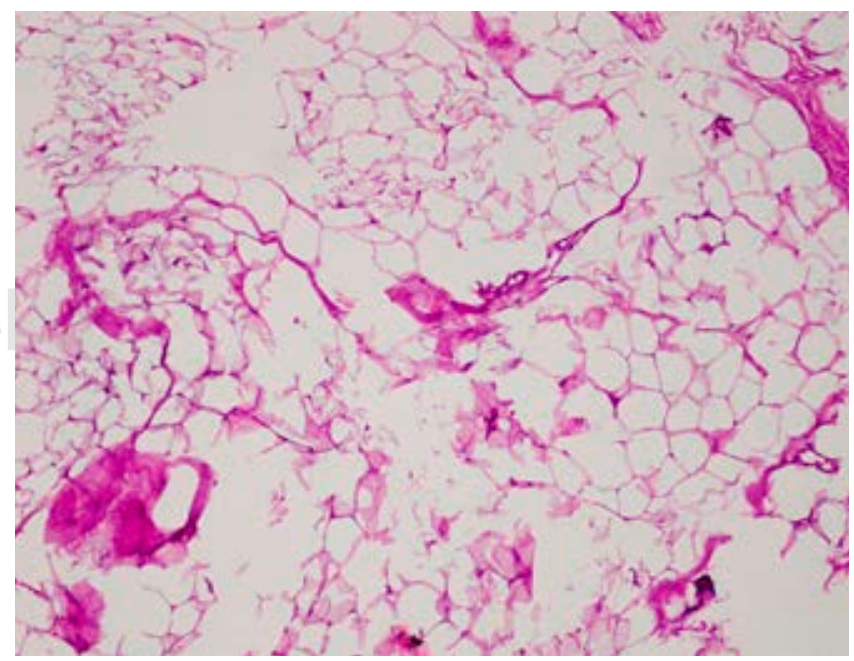

Figura 3: Histopatología de la lesión. 
tumores como lo son el sinovioma o fibrosarcomas, que no sólo requieren de un procedimiento quirúrgico más complejo, sino de un tratamiento multidisciplinario, compuesto por un cirujano oncólogo y un oncólogo clínico.

\section{CONCLUSIÓN}

Los fibrolipomas intermusculares son tumores benignos extremadamente raros. Para realizar un diagnóstico preciso las técnicas de imagen avanzadas (TAC/ RMN) son indispensables, además de que nos permiten una adecuada planeación preoperatoria para la resección de la lesión.

Bajo ciertas indicaciones, la extirpación quirúrgica es el tratamiento de elección, ya que proporciona un diagnóstico definitivo. Recomendamos una resección completa de las lesiones, para de esta forma evitar recurrencias y la necesidad de una reintervención quirúrgica.

\section{REFERENCIAS}

1. Charifa A, Azmat CE, Badri T. Lipoma pathology. In: StatPearls [Internet]. Treasure Island (FL): StatPearls Publishing; 2020.

2. Johnson CN, Ha AS, Chen E, Davidson D. Lipomatous softtissue tumors. J Am Acad Orthop Surg. 2018; 26 (22): 779788.
3. Liu DR, Li C, Chen L. Management of giant intermuscular lipoma of hips: A case report and review of literature. Mol Clin Oncol. 2013; 1 (2): 369-372.

4. Silistreli OK, Durmus EU, Ulusal BG, Oztan Y, Gorgu M. What should be the treatment modality in giant cutaneous lipomas? Review of the literature and report of 4 cases. $\mathrm{Br} \mathrm{J}$ Plast Surg. 2005; 58 (3): 394-398.

5. Echenique-Elizondo M. Intermuscular lipoma. J Am Coll Surg. 2001; 193 (4): 452.

6. Hakim E, Kolander Y, Meller Y, Moses M, Sagi A. Gigantic lipomas. Plast Reconstr Surg. 1994; 94 (2): 369-371.

7. Kajihara M, Sugawara Y, Sakayama K, Abe Y, Miki H, Mochizuki T. Subcutaneous fibrolipoma in the back. Radiat Med. 2006; 24 (7): 520-524.

8. Fletcher CD, Martin-Bates E. Intramuscular and intermuscular lipoma: neglected diagnoses. Histopathology. 1988; 12 (3): 275-287.

9. Gaskin CM, Helms CA. Lipomas, lipoma variants, and welldifferentiated liposarcomas (atypical lipomas): results of MRI evaluations of 126 consecutive fatty masses. AJR Am J Roentgenol. 2004; 182 (3): 733-739.

10. Kransdorf MJ, Bancroft LW, Peterson JJ, Murphey MD, Foster WC, Temple HT. Imaging of fatty tumors: distinction of lipoma and well-differentiated liposarcoma. Radiology. 2002; 224 (1): 99-104.

11. Nishida J, Morita T, Ogose A, Okada K, Kakizaki H, Tajino $\mathrm{T}$ et al. Imaging characteristics of deep-seated lipomatous tumors: intramuscular lipoma, intermuscular lipoma, and lipoma-like liposarcoma. J Orthop Sci. 2007; 12 (6): 533-541.

12. Kaeser MA, Smith LW, Kettner NW. A case report of an intermuscular lipoma: presentation, pathophysiology, differential diagnosis. J Chiropr Med. 2010; 9 (3): 127-131.

13. Serpell JW, Chen RY. Review of large deep lipomatous tumours. ANZ J Surg. 2007; 77 (7): 524-529. 